\title{
Exploring the lived experiences of first-time mothers and their partners affected by post-natal depression in rural Sanyati district, Zimbabwe
}

\author{
Mathew Nyashanu ${ }^{1 *}$, Thamary Karonga ${ }^{2}$, Fungisai Mushawa ${ }^{3}$
}

\begin{abstract}
${ }^{1}$ Department of Health and Allied Professions Nottingham Trent University, Nottingham, United Kingdom
${ }^{2}$ Department of Nursing and Midwifery Northrise University, Ndola, Zambia

${ }^{3}$ Department of Health and Social Work Nottingham, United Kingdom
\end{abstract}

Received: 07 May 2020

Accepted: 01 June 2020

\section{*Correspondence:}

Dr. Mathew Nyashanu,

E-mail: mathewnyashanu@ntu.ac.uk

Copyright: () the author(s), publisher and licensee Medip Academy. This is an open-access article distributed under the terms of the Creative Commons Attribution Non-Commercial License, which permits unrestricted non-commercial use, distribution, and reproduction in any medium, provided the original work is properly cited.

\begin{abstract}
Background: First time mothers and their partners are faced with a number of challenges including learning new skills and changing roles to meet the baby's needs. support the new baby. Although an exciting time, the early days of first-time parents is filled with excitement as well as challenges in adapting their lifestyle resulting in pressure and anxiety to look after the baby. The objective of this study was to explore the lived experiences of first-time mothers and their partners affected by post-natal depression (PND) among rural communities.

Methods: A collaborative exploratory qualitative approach underpinned by interpretive phenomenological analysis (IPA) was employed. Semi-structured interviews were conducted on ten (10) first time mothers and their partners who came for six-week post-natal care and for growth monitoring up to 6 months post-delivery at Sanyati Baptist hospital. Data were analysed using a thematic approach and guided by the four stages of data analysis in interpretive phenomenological analysis (IPA). N-vivo was used to organize the data to enhance management during analysis.

Results: The study showed that first time parents from rural communities felt trapped with childbirth, overwhelmed by changes, resultant disruptive relationships, lack of support and bouts of depression.

Conclusions: There is need to improve maternal support for first time mothers in rural communities. First time fathers need to learn new skills and adapt in supporting the baby and mother to prevent depression and marital breakdown.
\end{abstract}

Keywords: Biopsychosocial, Emotional support, First time parents, Post-natal depression

\section{INTRODUCTION}

Becoming a father or a mother for the first time is a significant transition in a man or woman's' life. The way this is managed may automatically affect the biopsychosocial-being of first-time parents in dealing with the new challenges of being a father or a mother. ${ }^{1}$ It becomes worse in situations where the traditional support systems are undergoing social and socio-economic change. First time parents have a challenge of learning new significant skills relating to infant care while recovering physically and emotionally from the effects of childbirth and associated social challenges. ${ }^{2}$ This takes place against a background of socio-economic hardships typical of a rural setting in a developing country like Zimbabwe. Becoming a parent for the first time can also cause anxiety with change of roles from looking after oneself to being responsible for the care of a new baby and considering the needs of one's partner.

As much as it brings happiness to first time parents, the new-born can also pose challenges to the young inexperienced couple. ${ }^{3}$ The baby comes with extra demands that can strain or limit the ever happy and vibrant nights out that the young parents were used to in rural Zimbabwe. ${ }^{4}$ The situation worsens when part of 
their disposable income has to meet the needs of the newly born baby. Furthermore, the situation can be bleak due to the poor economic outlook prevalent in these communities and failure of the first-time father to adequately provide for the family. ${ }^{5}$ The arrival of the baby also has a psychological impact on the parents. Family dynamics evolve with attention now focused on the baby depriving each other of the usual contrary to the life without the baby. ${ }^{6}$ Many first-time parents start to experience a decrease in marital satisfaction with the coming of the first child. ${ }^{7}$

A lot of young parents do not realise how life changing this transition can be until a few months into it and things become less realistic coupled with strained relationships full of blame and unhappiness. ${ }^{8}$ The new life comes with lack of sleep or sex and conflict over new roles. ${ }^{9}$ Such stressors are commonly shared by new parents across many communities. A 2012 study conducted by the essential parent company cited by ${ }^{10}$, showed that, 'around $80 \%$ of new parents felt both anxious and completely unprepared with the practical skills they need to look after their new baby.' Marital experts affirm that these problems are surmountable however, they can be alleviated through communication. ${ }^{11}$ Research has shown that first time parents who nurture their friendship with one another in the early days of the baby are likely to pull though the matrimonial challenges. This is so because it allows them to acclimatise and adopt to the new changes.

In many traditional rural communities immediate parents of first time fathers and mothers alongside their extended family normally provide immediate support which often cushions the turbulent times of first time parents. ${ }^{12}$ However, such support in traditional rural communities is being hampered by the ever-changing economic outlook that has seen relatives dispersing across the world in search of better life. This has left the enormous challenges of newly parenthood solely to inexperienced young parents with little or no support at all.

In light of the above assertion this research study explored the lived experiences of first-time mothers and their partners in copying with post-natal depression in a country undergoing social and economic challenges.

\section{METHODS}

The research study employed the exploratory qualitative approach underpinned by Interpretive Phenomenological Analysis (IPA). The data was analysed using a thematic approach and guided by the four stages of data analysis in (IPA). N-vivo was used to organize the data to enhance management during analysis. Ten first mothers and their partners were selected using purposive sampling. Semistructured interviews were conducted in person. Inclusion criteria was that women had given birth to a baby in the past six months and experienced emotional difficulties at some point during this period. Both parents were supposed to be first time mothers or fathers. Interviews were conducted over a period of six months using semistructured questions.

\section{Analysis}

The data was analysed using a thematic approach and guided by the four stages of data analysis in interpretive phenomenological analysis (IPA). ${ }^{13} \mathrm{~N}^{*}$ vivo was used to organize the data and enhance management during analysis. ${ }^{14}$

The analysis followed a four-stage process as follows:

In step (1) of the analysis, transcripts were read repeatedly to identify accounts of experiences that were important to the interviewee. The emic phenomenological position employed here concerned hearing and understanding the participant's story in their own words and keeping their experience at the centre of their account. In step (2), the etic phase, the accounts identified were re-read and pertinent sections summarized and given codes representing the researcher's interpretation. Steps 1 and 2 reflect the 'double hermeneutic' aspect of IPA whereby the participant interprets their own life experience and the researcher further interprets the participant's account. Step (3) involved identifying how these codes clustered together into themes and how themes were related to each other. Interviews were coded on a case-by-case analysis and themes labelled using key words and phrases from participants where possible to retain an idiopathic approach. In step (4) comparisons were made across the body of interviews to determine how prevalent themes were and how important they were to interviewees. The authors gave priority to the interviewees' accounts rather than their own personal and professional knowledge of experiences of pregnancy, birth and postnatal period. The results section provides descriptions of these themes, using verbatim quotes in support of themes.

\section{Ethical consideration}

Permission to conduct the study was sought from the medical research council of Zimbabwe and the Medical Superintendent of Sanyati Baptist Hospital. All research participants were given an information sheet and had the opportunity to ask questions prior to taking part in the study. The research participants also signed a consent form which gave them the right to withdraw from the study at any time without giving reasons. The research participants were also given information on where they could obtain support if they become affected by taking part in the research.

\section{RESULTS}

The following four themes were identified "feeling trapped, feeling overwhelmed, disrupted relationships, lack of support and feeling bouts of depression. 
Table 1: Themes and sub-themes identified in interviews.

\begin{tabular}{|ll|}
\hline Theme and subthemes & $\mathbf{N}(\%)$ \\
\hline Feeling trapped with childbirth & $10(50 \%)$ \\
\hline Negative feelings about birth & $9(45 \%)$ \\
\hline Guilt and self-blame & $8(40 \%)$ \\
\hline Poor support & $10(50 \%)$ \\
\hline Feeling overwhelmed & $20(100 \%)$ \\
\hline Increased responsibilities & $20(100 \%)$ \\
\hline Poor economic outlook & $8(40 \%)$ \\
\hline Inexperience & $20(100 \%)$ \\
\hline Disruptive relationship & $16(80 \%)$ \\
\hline No control over baby & $20(100 \%)$ \\
\hline No employment/ drought & $16(80 \%)$ \\
\hline Domestic violence & $16(80 \%)$ \\
\hline Lack of support & $20(100 \%)$ \\
\hline Needing and seeking support & $19(100 \%)$ \\
\hline Lack support from state institutions & $20(100 \%)$ \\
\hline Lack of support from relatives & $20(100 \%)$ \\
\hline Feeling bouts of depression & $20(100 \%)$ \\
\hline No hope for the future & $20(100 \%)$ \\
\hline Feeling pressurised to do well & $20(100 \%)$ \\
\hline Feeling embarrassed for failing & $20(100 \%)$ \\
\hline
\end{tabular}

\section{Theme 1: Feeling trapped with childbirth}

Nearly all the first-time mothers who took part in the study felt trapped following the birth of the child. They reported spending most of their time alone during the day and lack of friends to come and support them. Nearly all the first-time fathers who took part in the research study acknowledged poor support from relatives and had a feeling of being trapped in the new relationship. (see associated ratings of problems from research participants - Table 1.

"I wanted a baby boy, to please my husband. I am now regretting; this is a heavy load for me to carry. I thought the baby was going to bring me closer to my husband, but he has become more aggressive. I work very hard with my baby on the back to have food on the table while he does nothing. It is very painful to be a mother; my friends are no longer coming to see me" A 19-year-old first time mother.

"Honestly I feel depressed and trapped in this relationship I can't get any help no one comes to visit me I honestly didn't know that this is how new fathers feel given the chance I wouldn't do it" A 21-year-old first time father.

\section{Theme 2: feeling overwhelmed}

Both fathers and mothers in this research study complained of being overwhelmed by being first time parents. They reported increased responsibilities, a poor economic outlook, and lack of experience to issues that affected their parenthood. (see associated ratings of the issues in Table 1).

"I couldn't finish school because of the pregnancy, I feel guilty that I offended my parent. Although my husband is hardworking and loving, he comes home empty-handed after being absent for a month or more. There are no jobs the country has political and economic problems. I am afraid to lose my baby due to malnutrition, he is losing weight, we can afford one meal a day, the baby is now 6 months, I was told by the midwives that I should start giving other foods in addition to breast milk. The responsibilities are just too much" An 18-year-old first time mother.

"I can't get a job anywhere everyone is unemployed and survival is getting tough. It was better when I was living on my own, the responsibilities are too much I really feel overwhelmed by the coming of the baby. I am really failing as a father" A 19-year-old first-time father.

\section{Theme 3: disrupted relationships}

All the first-time fathers and mothers who took part in this research study reported disruption of relationships due to having no control over their own baby, unemployment, drought, and domestic violence. The first-time parents reported unilateral decisions from elders on the needs and health of the baby. They also complained of arguments caused by unemployment and shortage of food due between themselves due to unemployment. (see associated ratings of the issues in Table 1).

"When the baby is not feeling well, the decision to take the baby to traditional healers is made by the parents without our involvement. The unemployment and drought make it hard to feed the baby and we are always quarrelling with my partner over the problems blaming each other, honestly it is a relationship full of disruptions" A 20-year-old first time mother.

"I am still treated like a child the decision concerning the baby are made by parents. Authors are always quarrelling with my partner because I don't work and I am not bringing anything home. There is nothing in the field because of drought. Sometimes I blame myself" A 20year-old first time father

\section{Theme 4: lack of support}

Both first time fathers and mothers who took part in this research study reported lack of support from both the community and state institutions. The participants reported that the local clinic and relevant government institutions lacked programmes and support for first time parents in rural areas. They also reported that their elders had no time to teach them things as they were busy with their own lives/issues. (see associated ratings of the issues on Table 1). 
"The elders nowadays have no time to teach us young people about raising a family. It's all guessing game. Even the local clinic and other relevant government institutions have no supporting programmes for first time parent like us. A 21-year-old first time mother.

"Honestly, there is no help for first time fathers like me. The local clinic is clueless, no programmes to support first time parents like us. The government has nothing to offer for rural people. Honestly we need help" A 21-yearold first time father.

\section{Theme 5: feeling bouts of depression}

Both first time fathers and mothers who took part in this research study reported occasional feelings of depression, pressurised, hopeless and embarrassment for the failure to support the baby. (see associated ratings of the issues in Table 1).

"I sometimes have moments of depression when I think about my baby and my life. I feel hopeless and embarrassed because I can't support my only and first baby in the face of my community. Sometimes I wish I did not have my little one." An 18-year-old first time mother.

"I sometimes have long days of depression when I think about my failure to support my family as expected in my culture. I see myself as hopeless and at times so pressurised with problems. I hope this will come to an end and have a better future" A 19-year-old first time father.

\section{DISCUSSION}

Feeling trapped with childbirth normally happen with first time parents as they learn to spend more time at home with the baby as opposed to hanging out with friends. ${ }^{15}$ The findings suggest that first time mothers and fathers felt trapped with childbirth experiences as they spent most of their time working out modalities to support the new baby. However, good experiences about childbirth can be achieved if all new parents are supported to follow the new antenatal care guidelines of 8 or more antenatal care contacts whose aim is to promote good childbirth experiences from the antenatal period up to delivery throughout the post-natal period. ${ }^{16}$ This is supported by who cited that, the challenge of being trapped in childbirth could be circumvented by improving women's child birth experiences to decrease the likelihood of postpartum anxiety. ${ }^{17}$ Such support systems need the good will and material support from the central government to local authorities including hard to reach areas in remote parts of the country. Different support levels health systems in different locations is not new in low- and middle-income countries (LMICs) like Zimbabwe with rural areas affected by depleted health service system. ${ }^{18}$ There is need to balance health service improvement in urban areas and rural areas.
New parents normally feel overwhelmed by the coming of a new baby as the pace of life coupled with changing roles take toll on the first-time parents. ${ }^{19}$ Both the firsttime mothers and fathers reported being overwhelmed by parenthood responsibilities. Most of the research participants felt that parents and significant others in the community needed to support them in understanding issues affecting first time mothers and their partners including providing them with physical and emotional support where possible. It is important that first-time parents are enabled to access support and help from formal supporting systems outside their relationships. ${ }^{20}$ Such help can be provided through health professionals stationed at health facilities in the rural areas. It is therefore important that the central government through the Ministry of health makes such help and support available at designated points within rural health facilities. Alternatively, due to shortage of maternal professionals in many LMICs mobile health facilities can be rolled out in different rural settings for first time parents to access help and support. ${ }^{21}$ This can help to reduce the feelings of distress associated with feelings of being overwhelmed among first time parents in a remote rural setting.

Disruption of relationship in first time parents can be caused by a myriad of problems due to inexperience and poor supporting systems. ${ }^{22}$ The first time fathers and mothers in this study reported disruptive relationship due to poor support, unemployment and the impact of drought resulting in them struggling to have adequate food for the baby. In some instances, first-time mothers cited experiencing pain from the incision site. This in turn added pressure on the mother who was expected to carry maternal responsibilities whilst in agony. Failure to produce milk was also a stressor since the baby would be crying often and the mother struggling to rest. This is supported by who stated that most mothers have problems with breastfeeding due to stress. ${ }^{23}$ If the stress is not resolved early, it can end up causing depression, the most frequent psychiatric disorder which has longterm compromising effects on the mother-infant relationship and the child's development. ${ }^{24,25}$ Noted that there is growing evidence that anxiety is present among first-time mothers. This also leads to low maternal selfconfidence. According to, lack of self-confidence may damage early mother-infant relationship and negatively influence infant development. ${ }^{26}$ Some data suggest the same is true for men. ${ }^{27}$ Hard work in search for money to support the new family can result in aggression and abusive behaviour for first time fathers as highlighted that parental stress is also associated with postnatal depression. ${ }^{28}$ Symptoms of depression in fathers may manifest as low self-esteem, hostility, conflict, and anger. Fathers suffering from depression may withdraw or engage in "escape activities "such as overwork, gambling and excessive drinking. ${ }^{29}$ There is need for the central government through the Ministry of health and child welfare to establish early parental supporting systems for first time parents to mitigate disruption relationships. 
Such services can be effectively supported by community health workers as a way to mitigate shortage of qualified personnel. Community health workers have played a very important role in sustaining the health delivery of many LMICs. Such an initiative is viable and cost effective for developing countries under economic stalemate such as Zimbabwe.

Bouts of depression are also common characteristics of troubled first-time fathers and mothers. ${ }^{30}$ The research participants reported moments of depression due to feeling pressurized, having no hope and embarrassment due to failure in supporting the baby. In many African communities, fathers are expected to support their family and failure to do so is viewed as a life time failure through the lens of traditions and culture. ${ }^{31}$ Such a situation can lead to stress on the part of the father. Studies conducted by other researchers revealed that anxiety was the most common postnatal mental health problem in both men and women especially during the first six months of birth of their first infants. ${ }^{32}$ There is need to have established mental health workers working alongside maternal health professionals to support first time parents who may be affected by post-natal depression. More importantly there is need to support young families with start-up food packages that include baby foods with high nutrition for the baby's healthy development. Initiatives like this are not new in developing countries as they have been used following peace establishment in former conflict zones for young families. ${ }^{33}$ Such support can mitigate pressure among first time parents and provide an opportunity to explore other activities that can improve their health and well-being.

\section{Implications for practice}

There is need to improve maternal services for first time mothers and their partners in rural areas to make sure that first time parents are supported. More importantly community health workers need to be trained in supporting people affected by mental health to provide the first mental health safety net for rural communities especially first-time parents who might be prone to mental health problems due to the challenges they face. There is need for governments in developing countries such as Zimbabwe to decentralize health facilities to make sure that rural communities have up to date health facilities catering for a wide range of needs.

Traditional safety nets also need to be supported in enabling first time mothers and fathers to cope with the changes following the arrival of their baby. Previously, following the birth of a baby, a first-time mother would stay with their birth mother where they were supported to bring up their baby until 6 months of age.

Limitations of the study was carried out in one district in Zimbabwe; however, in future research conducted at a larger scale in several districts can provide generalizable findings which are key to health development and planning. This research mainly used qualitative approach. A research study using both quantitative and qualitative paradigms will need to be undertaken to enhance exploration of different issues utilizing different epistemological and ontological positions.

\section{CONCLUSION}

There is need to provide quality maternal health care for first time mothers and train first time fathers to positively support their partners in raising the baby. More importantly rural health facilities need to provide a multidisciplinary service to cater for all the needs of first-time mothers and their partners. First time mothers and fathers could benefit from through adopting a model utilised in Western countries, where health visitors monitor, for example, the UK, where health visitors monitor and give support to first time parents following the birth of their child. According to the Institute of Health Visiting in the UK, health visiting provides a platform through which individuals can be supported taking into account their specific needs. Recognising the limitations of the provision of such a qualified professional in Zimbabwe, this role could be fulfilled by the community health workers.

\section{ACKNOWLEDGMENTS}

Authors would like to thank all first-time mothers and their partners from Sanyati District who participated in this study.

\section{Funding: No funding sources}

Conflict of interest: None declared

Ethical approval: The study was approved by medical research council of Zimbabwe and the Medical Superintendent of Sanyati Baptist Hospital

\section{REFERENCES}

1. Ahlborg T, Strandmark M. The baby was the focus of attention-first-time parents' experiences of their intimate relationship. Scandinavian $J$ Caring Sci. 2001;15(4):318-25.

2. Emmanuel J, Simmonds S, Tyrer P. Systematic review of the outcome of anxiety and depressive disorders. The Br J Psyc. 2008;173(S34):35-41.

3. Madsen SA, Juhl T. Paternal depression in the postnatal period assessed with traditional and male depression scales. J Men's Health Gender. 2007;4(1):26-31.

4. Matthey S. Detection and treatment of postnatal depression (perinatal depression or anxiety). Current Opinion Psych. 2004;17(1):21-9.

5. Agénor PR, Canuto $O$. Gender equality and economic growth in Brazil: a long-run analysis. J Macroeconomics. 2015;43:155-72.

6. Parfitt Y, Ayers, S. Transition to parenthood and mental health in first-time parents. Infant Mental Health J. 2014;35(3):263-73

7. Ruthven I, Buchanan S, Jardine C. Isolated, overwhelmed, and worried: young first-time mothers 
asking for information and support online. J Associat Info Sci Technol. 2018;69(9):1073-83.

8. Saltzburg S. Narrative therapy pathways for reauthoring with parents of adolescent's coming-out as lesbian, gay, and bisexual. Contemp Family Ther. 2007;29(1-2):57-69.

9. Martyn E. Baby shock!: Your relationship survival guide. Random House; 2001. Available at: https://books.google.co.in/books/about/Baby_Shock.ht $\mathrm{ml}$ ?id $=16 \mathrm{kNswd} 8 \mathrm{eM} 8 \mathrm{C} \& \mathrm{redir}-\mathrm{esc}=\mathrm{y}$. Accessed on $14^{\text {th }}$ March 2020.

10. Winston R, Chicot R. The importance of early bonding on the long-term mental health and resilience of children, London J Prim Care (Abingdon) 2016;8(1):12-4.

11. Gipson JD, Hindin MJ. 'Marriage means having children and forming your family, so what is the need of discussion?' Communication and negotiation of childbearing preferences among Bangladeshi couples. Culture Health Sexual. 2007;9(2):185-98.

12. Abendroth AK, Van der Lippe T, Maas I. Social support and the working hours of employed mothers in Europe: the relevance of the state, the workplace, and the family. Social Sci Res. 2012;41(3):581-97.

13. Callary B, Rathwell S, Young BW. Insights on the process of using interpretive phenomenological analysis in a sport coaching research project. Qualitative Report. 2015;20(2):63-75.

14. Gale NK, Heath G, Cameron E, Rashid S, Redwood S. Using the framework method for the analysis of qualitative data in multi-disciplinary health research. BMC Med Res Methodol. 2013;13(1):117.

15. Fägerskiöld A. A change in life as experienced by firsttime fathers. Scand J Caring Sci. 2008;22(1):64-71.

16. World Health Organization. WHO recommendations on antenatal care for a positive pregnancy experience: summary: highlights and key messages from the World Health Organization's 2016 global recommendations for routine antenatal care. World Health Organization; 2018.

17. Bell AF, Carter CS, Davis JM, Golding J, Adejumo O, Pyra M, et al. Childbirth and symptoms of postpartum depression and anxiety: a prospective birth cohort study. Arch Women's Mental Health. 2016;19(2):21927.

18. Atiyeh BS, Gunn SWA, Hayek SN. Provision of essential surgery in remote and rural areas of developed as well as low- and middle-income countries. Int J Surg. 2010;8(8):581-5.

19. Ruthven I, Buchanan S, Jardine C. Isolated, overwhelmed, and worried: Young first-time mothers asking for information and support online. J Associat Info Sci Technol. 2018;69(9):1073-83.

20. Mbekenga CK, Christensson K, Lugina HI, Olsson P. Joy, struggle and support: postpartum experiences of first-time mothers in a Tanzanian suburb. Women Birth. 2011;24(1):24-31.

21. Hearn L, Miller M, Fletcher A. Online healthy lifestyle support in the perinatal period: what do women want and do they use it?. Aust J Prim Health. 2013;19(4):313-8.

22. Copeland D, Harbaugh BL. Differences in parenting stress between married and single first-time mothers at six to eight weeks after birth. Issues Comprehens Pediatr Nurs. 2005;28(3):139-52.

23. Henderson JJ, Evans SF, Straton JA, Priest SR, Hagan R. Impact of postnatal depression on breastfeeding duration. Birth. 2003;30(3):175-80.

24. Tronick E, Reck C. Infants of depressed mothers. Harvard Rev Psych. 2009;17(2):147-56.

25. Reck C, Struben K, Backenstrass M, Stefenelli U, Reinig K, Fuchs T, et al. Prevalence, onset and comorbidity of postpartum anxiety and depressive disorders. Acta Psychiatrica Scandinavica. 2008;118(6):459-68.

26. Reck C, Noe D, Gerstenlauer J, Stehle E. Effects of postpartum anxiety disorders and depression on maternal self-confidence. Infant Behav Develop. 2012;35(2):264-72.

27. Matthey S, Barnett B, Howie P, Kavanagh DJ. Diagnosing postpartum depression in mothers and fathers: whatever happened to anxiety? J Affect Dis. 2010;74:139-47.

28. Boyce P, Condon J, Barton J, Corkindale C. First-time fathers' study: psychological distress in expectant fathers during pregnancy. Aust New Zealand J Psyc. 2007;41(9):718-25.

29. Premberg A, Carlsson G, Hellström AL, Berg M. Firsttime fathers' experiences of childbirth-a phenomenological study. Midwifery. 2011;27(6):84853.

30. Beeghly M, Weinberg MK, Olson KL, Kernan H, Riley J, Tronick EZ. Stability and change in level of maternal depressive symptomatology during the first postpartum year. J Affect Dis. 2001;71(1-3):169-80.

31. Madhavan S, Townsend NW, Garey AI. 'Absent breadwinners': father-child connections and paternal support in rural South Africa. J Southern African Stud. 208;34(3):647-63.

32. Wynter K, Rowe H, Fisher J. Common mental disorders in women and men in the first six months after the birth of their first infant: a community study in Victoria, Australia. J Affect Dis. 2013;151(3):980-5.

33. Wessells M, Monteiro C. Supporting young children in conflict and post conflict situations: Child protection and psychosocial well-being in Angola. Africa's Future, Africa's Challenge. 2008:317.

Cite this article as: Nyashanu M, Karonga T, Mushawa F. Exploring the lived experiences of firsttime mothers and their partners affected by post-natal depression in rural Sanyati district, Zimbabwe. Int J Reprod Contracept Obstet Gynecol 2020;9:2759-64. 